\title{
A Comparison of the Growth and Asexual Reproduction by Cryphonectria parasitica Isolates Infected with Hypoviruses CHV3-County Line, CHV1-Euro7, and CHV1-Ep713
}

\author{
Jenise M. Bauman \\ Huxley College of the Environment, Western Washington University, Bellingham, Washington, D.C., USA \\ Email: Jenise.Bauman@wwu.edu
}

Received 3 December 2014; revised 18 December 2014; accepted 27 December 2014

Copyright (C) 2015 by authors and Scientific Research Publishing Inc.

This work is licensed under the Creative Commons Attribution International License (CC BY). http://creativecommons.org/licenses/by/4.0/ (c) (i)

\section{Abstract}

The impact of chestnut blight, caused by the fungal pathogen Cryphonectria parasitica, has diminished in Europe due to a natural biological control caused by hypovirus infection. Hypovirulence-mediated biological control has been far less successful in North America meriting further evaluation of field isolates that have the ability to produce non-lethal cankers, generate hypovirulent inoculum, and exhibit a greater ecological fitness in forest systems. In this study, Cryphonectria hypoviruses (CHV) CHV3-County Line, CHV1-Euro7, and CHV1-Ep713 were evaluated in five different isolates of $C$. parasitica. One hundred and eighty cankers representing each treatment combination were initiated on American chestnut sprouts in the Monongahela National Forest, West Virginia, USA. The size of cankers, the persistence of hypovirulent (HV) isolates, stroma production, and hypovirus transmission to conidia were assessed four and 12 months after canker expansion. CHV3-County Line infected isolates produced significantly smaller cankers than the isolates infected with either CHV1-type. With regard to CHV1-Euro7 isolates, the fungal genome appeared to contribute to the differences in canker size. After four months, HV isolates harboring either CHV1-type (30\%) were retrieved at a significantly higher rate than isolates containing CHV3-County Line (14\%). After 12 months, the HV recovery was similar among the three hypoviruses indicating smaller cankers will maintain their HV status after one year. Very few stroma were produced after one year in the field from $\mathrm{HV}$ isolates. In vitro, CHV3-County Line (49\%) had a significantly lower rate of hypovirus transmission to conidia when compared to CHV1-Euro7 (87\%) and CHV1-Ep713 (80\%). Significant differences existed among the five different isolates indicating HV transmission is dependent on the fungal genome. This research provided additional evidence that each hypovirus interacts with its host differently and certain isolate/hypovirus combinations 
have better biological control potential than others.

Keywords

American Chestnut, Biological Control, Chestnut Blight, Cryphonectria parasitica, Hypovirulence, Hypovirus

\section{Introduction}

The impact of chestnut blight, caused by the fungal pathogen Cryphonectria parasitica, has diminished in Europe presumably due to a natural biological control caused by hypovirus infection [1] [2]. Hypoviruses are viruslike particles of dsRNA found in the cytoplasm of debilitated strains of C. parasitica [3] [4]. Infected isolates display a significant reduction in fungal virulence (hypovirulence) coupled with reduced asexual spore production and sexual infertility. Cloning sequence analysis has allowed for closer examination of dsRNA, which often differs among Cryphonectria hypoviruses (CHV) with respect to size and number of coding regions [5]-[7]. Four types of dsRNA viruses (CHV1, CHV2, CHV3, and CHV4) have been described and placed in the genus Hypovirus in the family Hypoviridae [6] [8]-[10].

CHV1 hypoviruses have been released in many areas of North America but are found naturally occurring in infected C. parasitica isolates in Europe and parts of Asia [11]-[13]. CHV1-Ep713 is a CHV1-type hypovirus isolate with a $12.7 \mathrm{~kb}$ genome comprised of two coding regions designated ORF A and ORF B [8]. When compared to CHV1-Euro7, another European CHV1-type isolate, the level of nucleotide identity for the entire 5' non-coding domain is 93\%; the CHV1-Euro7 genome is 11 nucleotides shorter than the CHV1-Ep713 genome [14]. Despite the similarity, these two isolates have phenotypic and morphological traits that differ considerably [5]. CHV1-Ep713 is a severely debilitating hypovirus isolate in vitro and produces small cankers and few asexual spores in vivo. In contrast, CHV1-Euro7 infection has a milder impact on fungal virulence; this isolate grows rapidly in vitro and produces larger cankers in vivo with moderate asexual sporulation [14].

CHV3-type isolates are endemic to North America. Biological control with this hypovirus occurs naturally in chestnut stands in Michigan and has been recovered from experimental plots in West Virginia and Kentucky [15] [16]. CHV3-hypoviruses have a less effect on sporulation and pigmentation, however, significantly reduces the virulence of $C$. parasitica [17]. Typically, the vegetative growths of these isolates are severely debilitated, producing small, superficial cankers both in vivo and in vitro [16]. Previous studies have identified fundamental differences in the CHV3 dsRNAs when compared to CHV1 [18]. Sequencing results for hypovirulent isolate, CHV3-Grand Haven 2, have determined that this hypovirus is comprised of a single ORF and a $9.8 \mathrm{~kb}$ genome; considerably distinct from the two ORF, $12.7 \mathrm{~kb}$ genome organization of the CHV1 hypovirus [9] [17].

Despite the variation among hypovirus-containing strains, they all have the potential to arrest canker expansion, as noted in the callousing cankers in European and in North American orchards [2] [19] [20]. Hypoviruses can also be transmitted into asexual conidia producing hypovirulent inoculum in natural populations [21]. In addition, each hypovirus-type is transmissible in nature via anastomosis among the same vegetative compatibility (vc) types in C. parasitica populations [14] [22]. Hypovirulence-mediated biological control has been far less successful in North America than in Europe, presumably due to barriers caused by diversity in vc types [16] [23]. However, HV transmission has also been reported to occur between different vc types, although to a much lesser extent [24] [25]. This indicates that hypovirulence conversion between incompatible isolates may provide successful transmission into natural populations of C. parasitica overtime [13] [25] [26].

Field isolates that have the ability to produce non-lethal cankers, generate hypovirulent inoculum, and exhibit a greater ecological fitness are sought after to promote a successful biological control agent [12] [22]. The notion is that an isolate that has a greater tolerance to hypovirus-infection will produce a larger canker with the propensity to persist in the forest and produce hypovirus inoculum through viable asexual spores. Studies that analyze the interactions between the fungus and various hypoviruses have been conducted with the intention of selecting isolates that display a less debilitated growth habit after hypovirus acquisition [5] [14] [27]. In this present study, CHV1-Euro7, CHV1-Ep713, and CHV3-County Line hypoviruses were evaluated in five different strains of $C$. parasitica to determine the influences various hypoviruses have on different genomes of the 
fungus. Variables such as pathogenicity, HV persistence in the field, stroma production, and hypovirus transmission to conidia (in vivo and in vitro) were evaluated.

\section{Methods}

\subsection{Parasitica Isolates}

The 20 strains of $C$. parasitica that were used in this study are listed in Table 1. Five virulent isolates were used as controls: Bockenhauer, Schomber, Euro7ssv, JR10, and WR2. All virulent strains originally were isolated from natural cankers found on American chestnut with the exception of Euro7ssv. This virulent isolate originated from a single conidial isolation of hypovirulent isolate Euro7. The original $\mathrm{HV}$ isolate was obtained from a canker on European chestnut growing near Florence, Italy. The Bockenhauer and Schomberg virulent isolates were recovered from cankers on American chestnut growing near West Salem, WI. JR10 and WR2 were isolated from cankers in Front Royal., VA. Each hypovirulent isolate contained either hypovirus CHV1-Euro7, CHV1-Euro7, or CHV3-County Line hypovirus (Table 1; Figure 1). Each HV isolate, harbored only one hypovirus per isolate, was created in the laboratory by anastomosis-mediated transfer of hypovirus dsRNA from infected isolates to the virulent parent (M. Double, per comm.). All hypovirus transmissions were confirmed by phenol/chloroform extractions that show the presence of dsRNA bands along a gel gradient in the acquiring strain as described by Dodds (1978). All cultures were maintained on Potato Dextrose Agar (PDA) and stored at $20^{\circ} \mathrm{C}$ with a $16: 8$ hour photoperiod.

\subsection{Field Canker Initiation}

Inoculum used to initiate field cankers was prepared by transferring a small piece of mycelium from test tube stock cultures to the center of Petri plates containing PDA medium. Cultures were incubated for seven days at $20^{\circ} \mathrm{C} 58 \mathrm{~cm}$ beneath two 34 watt fluorescent lamps with alternating 16 hours of light and 8 hours of darkness. Cankers were initiated by hypovirulent strains of fungus that were applied four per tree at $50 \mathrm{~cm}, 100 \mathrm{~cm}, 150$

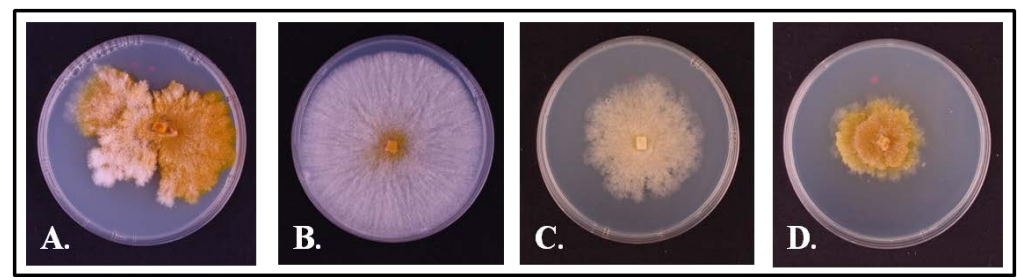

Figure 1. Examples of morphologies exhibited by virulent (A) and hypovirus infected isolates (B)-(D). Figure shows from left to right (A) Bockenhauer hypovirus-free; (B) Bockenhauer(CHV1-Euro7); (C) Bockenhauer(CHV1-Ep713); and (D) Bockenhauer(CHV3-County Line).

Table 1. Isolate combinations used in this study. Five virulent isolates were used as controls: Bockenhauer, Schomberg, Euro7ssv, JR10, and WR2. Each acquired one hypovirus type (CHV1-Euro7, CHV1-EP713, or CHV3-County Line) through anastomosis-mediated transfer of hypovirus dsRNA from infected isolates. The “+” indicates successful transfer of hypovirus into specific fungal strain after confirmation by phenol/chloroform extractions.

$\begin{array}{cccccc} & & & \\ & \text { C. parasitica fungal strains } & & \text { Euro7ssv } & \text { JR10 } \\ \text { Hypovirus } & \text { Bockenhauer } & \text { Schomberg } & + & + \\ \text { Virulent } & + & + & + & + \\ \text { CHV1-Euro7 } & + & + & + & + \\ \text { CHV1-Ep713 } & + & + & + \\ \text { CHV3-County Line } & + & + & + \\ +\end{array}$


$\mathrm{cm}$, and $200 \mathrm{~cm}$ above the ground. Fifty-seven blight-free American chestnut sprouts that ranged from six to nine centimeters in diameter were used for this study. The sprouts were in an area of the Potomac Ranger District of the Monongahela National Forest, West Virginia, which contained abundant coppice populations of American chestnut. The specific plot used for this research is on Big Mountain Road (USFS \#48) of the Snowy Mountain Quadrangle, located at $1119 \mathrm{~m}$ above sea level. The global position is: north 38 35.5'58.6"/west $79^{\circ} 35^{\prime} 25.5^{\prime \prime}$.

All trees were infected by removing a 7-mm bark disk using a sterilized 7-mm diameter cork borer and inserting a similar size piece of mycelium removed from the margin of seven-day-old cultures. To prevent more than one hypovirus hybridizing within the same thallus of a test canker, trees were first randomly designated by hypovirus, and then canker placement was randomly assigned per strain. To avoid hypovirus contamination of virulent cankers, the control trees (harboring virus-free isolates) were located $15 \mathrm{~m}$ from the nearest hypovirus-inoculated tree, buffered by a logging road and existing forest trees. Two virulent cankers were established at $50 \mathrm{~cm}$ and $150 \mathrm{~cm}$. The inoculation site was covered with tape to retard desiccation. All virulent and hypovirulent inoculations were replicated nine times for each isolate.

\subsection{Field Data Collection}

To determine growth rates, all cankers were measured (length and width $\mathrm{cm}$ ) after 12 months of field growth. Canker area was calculated from the radius $((\mathrm{L}+\mathrm{W}) / 2)$. Bark samples were collected to assess whether the test isolate retained its original infection status in the canker (HV recovery). Three bark samples were removed from the margin of each canker with a sterilized bone marrow biopsy instrument. The bone marrow biopsy instrument was sterilized between each sample. One sample was removed from the center of the upper margin of the expanding canker, the second sample was extracted from the bottom right margin, and the third sampled was taken from the bottom left margin. Bark samples were collected in 96-well micro-titer plates (Fisherbrand Scientific) and stored at $-20^{\circ} \mathrm{C}$.

To culture fungal isolates, bark plugs were transferred to a Petri dish containing glucose yeast extract medium (GYE) amended with antibiotics Tetracycline Hydrochloride (100 mg/L) and Streptomycin Sulfate (10 mg/L). Bark plugs were incubated at room temperature under natural light conditions for three-to-five days. The resulting mycelium was transferred to PDA and incubated at $20^{\circ} \mathrm{C}$ with a $16: 8$ hour photoperiod for 7 to 10 days. The morphology of the resulting colonies was analyzed to determine if the retrieved isolates appeared hypovirulent or virulent and if their growth, coloration, and margin formation were similar to parental isolates used to initiate the cankers (Figure 1).

\subsection{Analysis of Asexual Sporulation}

To assess hypovirus transmission to asexual conidia, single conidial cultures were obtained from the pycnidia of field cankers (in vivo) and from isolates cultured on PDA (in vitro). To do this, a small amount of conidial exudate was collected from bark samples and culture plates by a sterile spear tip and serially diluted in $0.1 \%$ peptone solution. Spore dilutions were aseptically transferred with a pipette onto the surface of GYE + antibiotics and streaked using a sterile glass rod. Plates were incubated at room temperature under natural lighting for fortyeight hours. Fifty individual germinating conidia (per canker) were located under a dissecting microscope and were aseptically transferred onto PDA using a sterile surgical blade ( 5 germlings per plate). Plates were incubated for seven-to-fourteen days at $20^{\circ} \mathrm{C}$ with a 16:8 hour photoperiod. Resulting colonies were scored as virulent or hypovirulent based on their cultural morphology. Three replications were provided per isolate.

\subsection{Statistical Analysis}

Hypovirus and $C$. parasitica strain combinations formed the 4X5 factorial design (Table 1). A mixed model, two-way analysis of variance (ANOVA) was used with fixed effects being hypovirus and parental background with trees as a random effect. Box plots illustrated a full comparison of all treatment combinations for multiple comparisons. HV transmission to conidia in vitro was compared per hypovirus parental background combination with a one-way ANOVA using Tukey's HSD as a post-hoc test. Log10 transformations were used to meet the assumptions of normality and unequal variances. Differences were considered significant when $P \leq 0.05$ according to the $F$ test. All statistics were analyzed using JMP version 5 (SAS Institute, Cary, North Carolina). 


\section{Results}

\subsection{Analysis of Canker Growth}

After 4 months, the average size $((\mathrm{L}+\mathrm{W}) / 2)$ of all virulent cankers was $7.0 \mathrm{~cm}$. After 12 months, the average size of virulent cankers had increased to $18.8 \mathrm{~cm}$. This was significantly larger than those produced by $\mathrm{HV}$ isolates (Figure 2). Results analyzed using a two-way ANOVA revealed a significant interaction between hypovirus by parental background $(P=0001)$. CHV3-County Line infected isolates produced significantly smaller cankers than isolates containing either of the CHV1-type hypoviruses (CHV1-Euro7 or CHV1-Ep713) $(P=0.0002)$. HV Bockenhauer and Schomberg isolates showed a greater variability in hypovirus effect, again illustrating significantly smaller cankers by CHV3-County Line isolates when compared to their CHV1 counterparts $(P=0.001)$. Euro7ssv, JR10, and WR2 isolates produced cankers similar in size regardless of the hypovirus they contained (Figure 2).

\subsection{Bark Sample Analysis to Evaluate Hypovirus Recovery}

The morphology of the isolates cultured from bark plugs removed from cankers formed the basis for virulence or hypovirulence (HV) determination. Fungi isolated from individual bark plugs extracted from all 4-month-old and 12-month-old cankers were scored as either percent virulent, non-C. parasitica, or HV (Figure 3). After four months, significant interactions between hypovirus and fungal strain were recorded $(P=0.004)$. Of these, Bockenhauer and Schomberg yielded a greater percent of HV. HV recovery increased after 12 months. The same trend occurred; significant interactions between hypovirus and parental background existed $(P=0.003)$ and greater percent of HV recovery from Bockenhauer and Schomberg initiated cankers occurred.

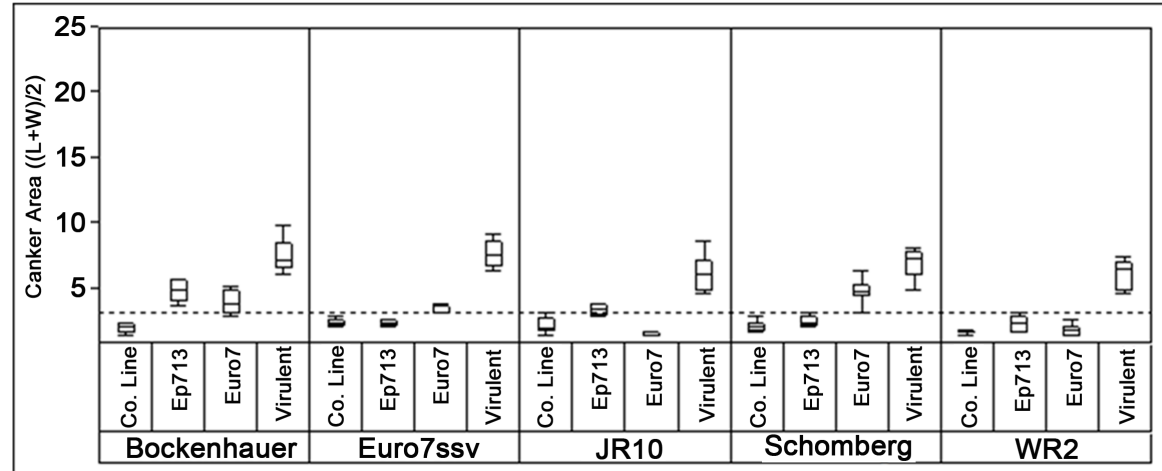

Canker Area: Comparison hypovirus by parental background after 4 months

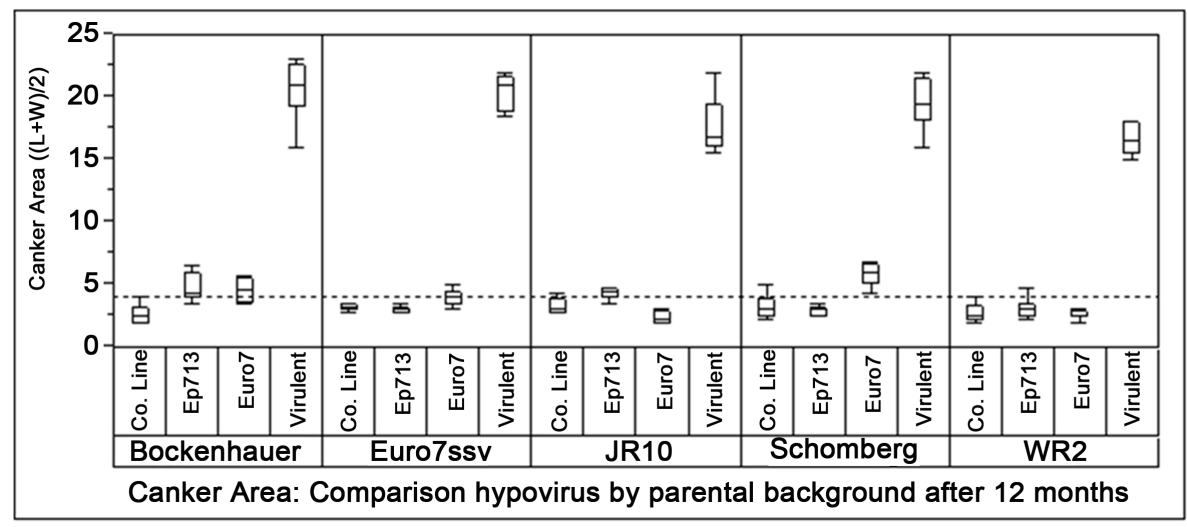

Figure 2. Comparison of CHV3-County Line (Co. Line), CHV1-Ep713 (Ep713), and CHV1Euro7 (Euro7) isolates with regard to canker size $((\mathrm{L}+\mathrm{W}) / 2)$. There was a significant interaction between the two factors $(P=0.0001)$. Box plots illustrate differences among hypovirus and $C$. parasitica strains. Virulent cankers were significantly larger and Co. Line cankers significantly smaller $(\mathrm{P}<0.05)$. 


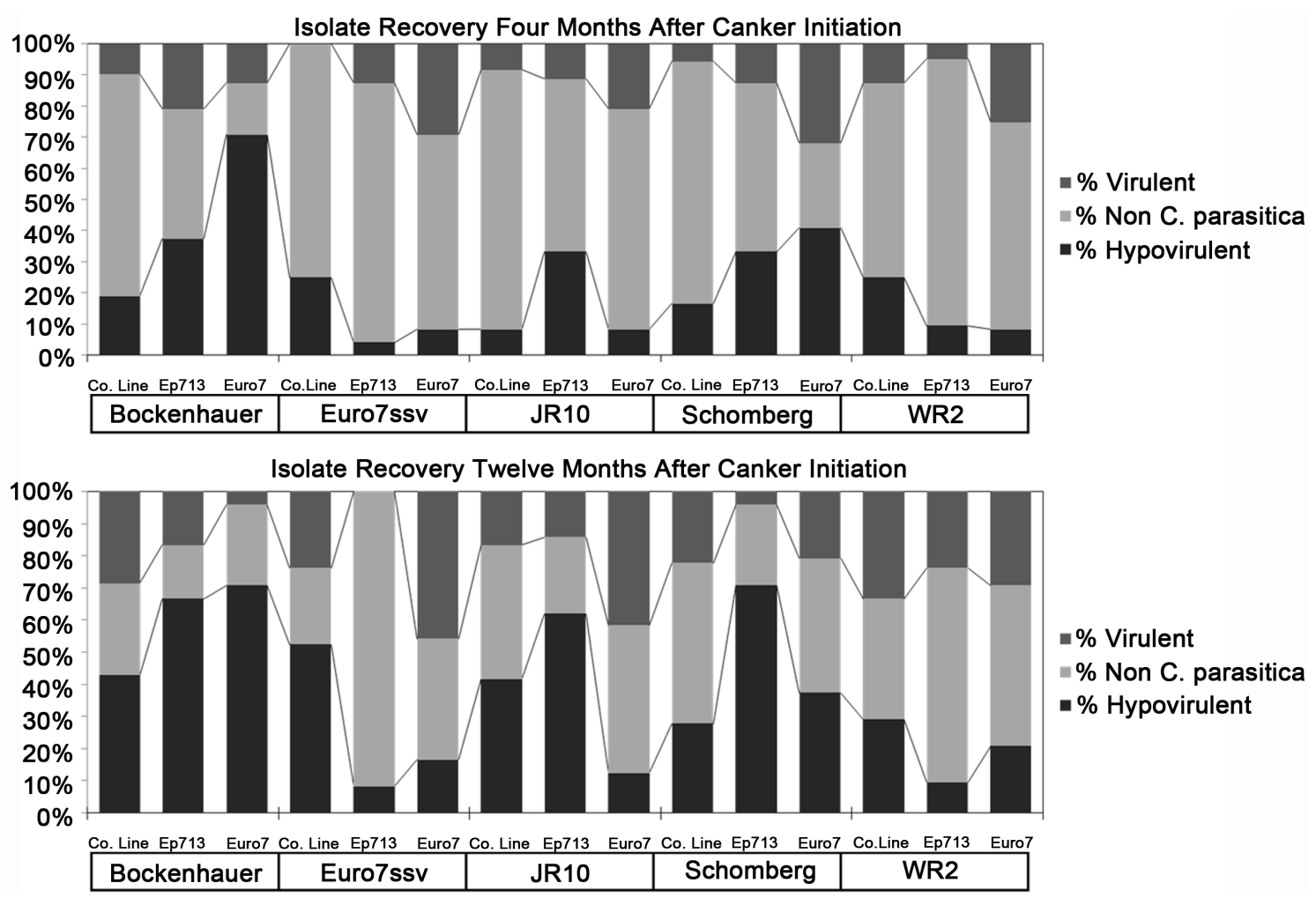

Figure 3. Comparison of CHV3-County Line (Co. Line), CHV1-Ep713 (Ep713), and CHV1-Euro7 (Euro7) hypoviruses in terms of percentage of hypovirulent (HV), virulent (V), and non-C. parasitica (Non Cp) isolates recovered from all cankers per parental background sampled after 4 and 12 months after initiation.

\subsection{HV Transmission from Conidia in Vivo}

The majority of the HV C. parasitica isolates used to initiate field cankers did not produce stroma. After four months, only 17 of $128 \mathrm{HV}$ isolates formed cankers that produced stroma (Table 2). Furthermore, of those 17 cankers, only 9 produced stroma with viable conidia. Of these, the Schomberg (CHV1-Euro7) isolate produced the highest amount of HV conidia (60\%). After 12 months, only $8 \mathrm{HV}$ cankers produced visible stroma (Table 3). Of these, Schomberg (CHV1-Euro7) and Bockenhauer(CHV1-Ep713) isolates were the better stroma producers of HV conidia ( $38 \%$ and $48 \%$, respectively; Table 3 ). Of the CHV3-infected isolates, Schomberg and Euro7ssv yielded HV conidia of $2 \%$ and $16 \%$, respectively (Table 3 ).

\subsection{Comparison of HV Transmission to Conidia in Vitro}

Test isolates produced stroma containing viable conidia when cultured on PDA in the laboratory. Germlings from asexual spores were scored either HV or V based on morphology in culture. There were significant 2-way interactions among the parental backgrounds and the three hypoviruses $(P=0.001)$. Transmission among the $C$. parasitica strains infected with CHV3-County Line was significantly lower, with the exception of WR2. Bockenhauer and Schomberg CHV1 isolates were consistent with high HV transmission rates. A greater variability existed in Euro7ssv, JR10, and WR2 (Figure 4).

\section{Discussion}

Characteristics thought to be a necessary for using hypoviruses as biological control agents include successful HV canker establishment, isolate persistence in the environment, and production of HV conidia. After 4 and 12 months in the field the influence of the hypovirus-infection on the growth of isolates was clearly demonstrated. The cankers initiated by virulent isolates produced significantly larger cankers than their HV counterparts. This finding was consistent with previous studies [27] [28]. With regard to CHV3-County Line hypovirus, isolates 
Table 2. The percentage of HV transmission to conidia from CHV3-County Line, CHV1-Euro7, and CHV1-Ep713 isolates sampled from 4-month-old field cankers. Table reports on the number of cankers that were producing stroma (\# of cankers), the number of the stroma bearing cankers that produced viable spores (viable stroma), and the percentage of HV colonies that cultured from asexual spores yielded from field canker stroma (\% HV conidia).

\begin{tabular}{|c|c|c|c|c|c|}
\hline \multicolumn{6}{|c|}{ Ratings of Asexual Reproduction After 4 months } \\
\hline CHV3-County Line & Bockenhauer & Schomberg & Euro7ssv & JR10 & WR2 \\
\hline \# of cankers & 0 & 0 & 3 of 7 & 1 of 6 & 0 \\
\hline viable stroma & 0 & 0 & 3 of 3 & 0 & 0 \\
\hline \% HV conidia & - & - & $8 \%$ & - & - \\
\hline CHV1-Euro7 & Bockenhauer & Schomberg & Euro7ssv & JR10 & WR2 \\
\hline \# of cankers & 2 of 8 & 6 of 8 & 1 of 8 & 0 & 0 \\
\hline viable stroma & 0 of 2 & 4 of 6 & 0 of 1 & 0 & 0 \\
\hline \% HV conidia & - & $60 \%$ & - & - & - \\
\hline CHV1-Ep713 & Bockenhauer & Schomberg & Euro7ssv & JR10 & WR2 \\
\hline \# of cankers & 1 of 5 & 0 & 0 & 0 & 0 \\
\hline viable stroma & 0 & 0 & 0 & 0 & 0 \\
\hline \% HV conidia & - & - & - & - & - \\
\hline
\end{tabular}

Table 3. The percentage of HV transmission to conidia from CHV3-County Line, CHV1-Euro7, and CHV1-Ep713 isolates sampled from 12-month-old field cankers. Table reports on the number of cankers that were producing stroma (\# of cankers), the number of the stroma bearing cankers that produced viable spores (viable stroma), and the percentage of HV colonies that cultured from asexual spores yielded from field canker stroma (\% HV conidia).

\begin{tabular}{|c|c|c|c|c|c|}
\hline \multicolumn{6}{|c|}{ Ratings of Asexual Reproduction After 12 Months } \\
\hline CHV3-County Line & Bockenhauer & Schomberg & Euro7ssv & JR10 & WR2 \\
\hline \# of cankers & 0 & 1 of 6 & 3 of 7 & 0 & 0 \\
\hline Viable stroma & 0 & 1 of 1 & 3 of 3 & 0 & 0 \\
\hline \% HV conidia & - & $2 \%$ & $16 \%$ & - & - \\
\hline CHV1-Euro7 & Bockenhauer & Schomberg & Euro7ssv & JR10 & WR2 \\
\hline \# of cankers & 1 of 8 & 1 of 7 & 0 & 0 & 0 \\
\hline Viable stroma & 1 of 1 & 1 of 1 & 0 & 0 & 0 \\
\hline \% HV conidia & $6 \%$ & $38 \%$ & - & - & - \\
\hline CHV1-Ep713 & Bockenhauer & Schomberg & Euro7ssv & JR10 & WR2 \\
\hline \# of cankers & 2 of 7 & 0 & 0 & 0 & 0 \\
\hline Viable stroma & 1 of 2 & 0 & 0 & 0 & 0 \\
\hline \% HV conidia & $48 \%$ & - & - & - & - \\
\hline
\end{tabular}

infected with this hypovirus produced significantly smaller cankers than the CHV1-hypoviruses when assessed after either 4 or 12 months of growth (parental backgrounds pooled). Furthermore, all fungal backgrounds produced cankers similar in size when infected with CHV3-County Line. Isolates that produced larger cankers were severely affected by the CHV3-County Line hypovirus. The significant debilitation caused by the CHV3-type hypovirus has been observed in previous studies [9] [16], presumably due to the significant down regulation of genes responsible for pathogencity. 


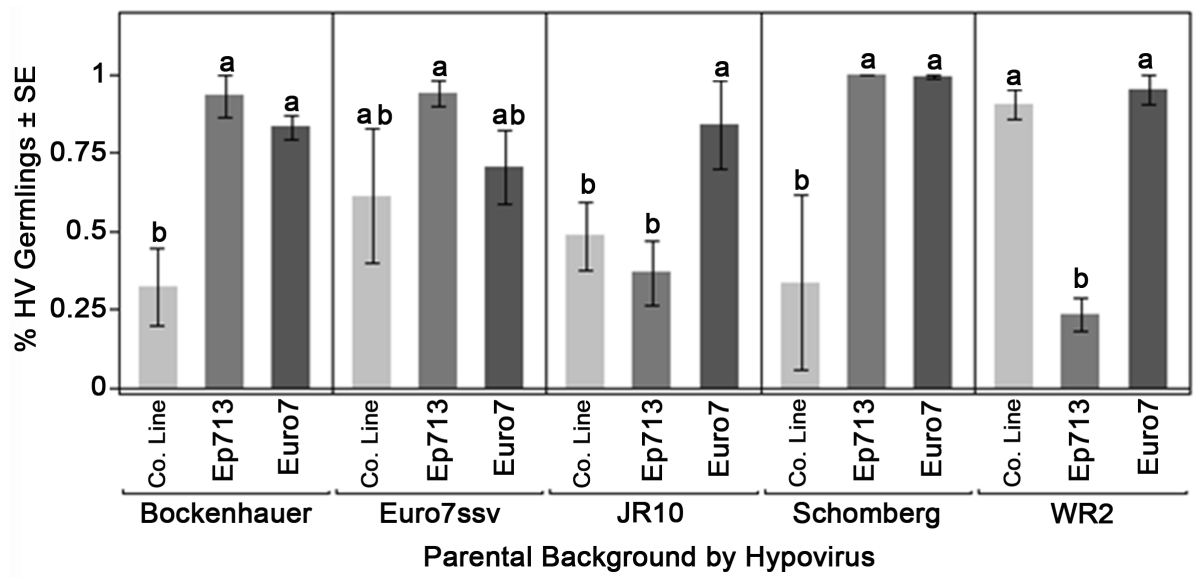

Figure 4. In vitro comparison of hypovirus transmission into conidia among the various test isolates (Bockenhauer, Euro7ssv, JR10, Schomberg, and WR2) infected with one of the three hypoviruses: CHV3-County Line (Co. Line), CHV1-Ep713 (Ep713), and CHV1-Euro7 (Euro7). Bars show the percentage of hypovirulent colonies resulting from single-conidial isolation. Differences existed among the different hypovirus, parental background combination $(P$ $=0.0001)$. Measurements sharing common letters do not significantly differ from each other (alpha $=0.05$ ) determined by Tukey’s HSD.

Variability existed among the fungal strains, as in other studies [12] [29]. The C. parasitica fungal strain appeared to contribute to the differences in the size of cankers produced by the CHV1-Euro7 infected isolates. The variations in rankings observed among the isolates infected with CHV1-Euro7 were in most cases similar to the rankings of the isolates in their virulent form. That is, the most invasive strains, Bockenhauer and Schomberg, generally produced, with some exceptions, significantly larger cankers. This would indicate that the fungal genome plays a very important role in CHV1 hypovirus expression and therefore, how well suited particular field isolates are as biological control agents [12]. When comparing CHV1-Ep713 to CHV1-Euro7 isolates, there were generally no significant differences in size of cankers produced when cankers were compared per fungal strain. This was in contrast to previous studies that identified isolate Ep155 more debilitated when infected with CHV1-Ep713 with regard to canker size and sporulation than CHV1-Euro7 isolates growing on excised chestnut stems [14] [30]. Euro7ssv isolates produced cankers similar in size regardless of the infecting CHV1 hypovirus. Isolates JR10 and WR2, when infected with the CHV1-Euro7, produced smaller cankers than their CHV1Ep713 counterparts.

As the cankers aged during this study, the percentage of hypovirulent isolates recovered increased. This occurred regardless of parental background or hypovirus. Cankers that enlarged the most (Schomberg, Bockenhauer, and Ep155) generally yielded the highest percentage of hypovirulent isolates. The recovery of the two CHV1-types was significantly higher than CHV3-County Line after 4 months in the field, consistent with the larger cankers yielding higher hypovirus recovery. However, after 12 months the HV recovery was similar among the three hypoviruses. Field studies have reported the persistence of CHV3 in West Virginia 12 years after initial establishment [15]. The natural establishment of CHV3 in Michigan may indicate a naturally suited hypovirus for the forests of North America [31].

Stroma production on field cankers initiated by HV isolates was poor compared to virus-free isolates, a common consequence to the attenuation in virulence caused by hypovirus infection reported in other studies [20] [28] [32] [33]. If the genes responsible for enzymatic activity in the fungus are down regulated due to hypovirus-infection [9] an HV isolate will lack the ability to activate enzymes required for tissue degradation, nutrient acquisition, and the formation of stroma. Stroma production in vivo may have been further diminished by host response of the chestnut tree with the defensive production of polyphenolic compounds [34], ethylene [35], and callous tissue. The production of hypovirulent conidia in the forest ecosystem helps maintain the natural spread of hypoviruses and would seem to be a requirement for successful biological control. During this field study, isolates that also were the most pathogenic (Bockenhauer and Schomberg) were HV cankers that yielded HV inoculum. 
Conidia formation as well as the percentage of hypovirus transmission to conidia was significantly higher in the laboratory than in the field. CHV3-County Line transmission percentages in vitro had a wider variation in rankings among parental backgrounds than those infected with CHV1-Euro7 or CHV1-Ep713. CHV1-infected isolates have been reported to transmit their hypoviruses at rates ranging from 90\% - 100\% [9] [16]. During this study CHV1-Ep713 and CHV1-Euro7 infected isolates had similar transmission rates averaging $80 \%$ - 87\%, respectively. An increase in HV conidia from the CHV1-type hypoviruses may be a function of the protein p40 found on ORF A on the genomes of CHV1 hypoviruses. This protein is associated with CHV1 hypoviruses may increase ORF B expression that in turn enhances viral replication and dsRNA accumulation in asexual spores [9]. There were a few exceptions among the CHV1 infected isolates where certain backgrounds generated unusually low hypovirus transmission rates; a trait noted in previous single-conidial isolations indicating the fungal genome may have some regulatory effects on how well the hypovirus is able to replicate and be passed on during conidiogenesis [28] [32].

\section{Conclusion}

In summary, this research provides additional evidence that each hypovirus interacts with its host differently. The results support previous research that reported varying morphological and pathogenic characteristics dependent on the genome of the isolate and hypovirus [14] [28] [32]. The most important message consequential to this study is that certain isolate/hypovirus combinations have better biological control potential than others. It would seem that those fungal isolates that are most pathogenic also have other traits such as increased ability to sporulate and high HV transmission rates that merit their use as biological control agents [22]. This, however, may not always be the case. For example, certain hypovirus/isolate combinations, JR10 and WR2 infected with CHV1-Euro7, performed very poorly in comparison to the other isolates infected with the same hypovirus. However, these isolates showed an improved biological control potential when harboring CHV1-Ep713. Therefore, hypovirus infection may not create strains that are uniform in all biological control characteristics. Rather, it appears to be a complex interaction between fungus and hypovirus genomes resulting in isolates with varying attributes.

\section{Acknowledgements}

Funding and research support for this project was provided by The American Chestnut Foundation and West Virginia University. Thank you to Dr. William MacDonald and Mark Double of West Virginia University for their guidance, contribution, and thoughtful feedback throughout this study. Author thanks Anthony Barker, Jessica Bright, and William Rittenour for their assistance in the field and laboratory procedures.

\section{References}

[1] Grente, J. and Berthelay-Sauret, S. (1978) Biological Control of Chestnut Blight. In: MacDonald, W.L., Cech, F.C., Luchok, J. and Smith, H.C., Eds., Proceedings of the American Chestnut Symposium, West Virginia University Press, Morgantown, 30-34.

[2] Robin, C. and Heiniger, U. (2001) Chestnut Blight in Europe: Diversity of Cryphonectria parasitica, Hypovirulence and Biocontrol. Forest Snow and Landscape Research, 76, 361-367.

[3] Dodds, J.A. (1980) Association of Type 1 Viral-Like dsRNA with Club-Shaped Particles in Hypovirulent Strains of Endothia parasitica. Virology, 107, 1-12. http://dx.doi.org/10.1016/0042-6822(80)90267-6

[4] Newhouse, J.R., Hoch, H.C. and MacDonald, W.L. (1983) The Ultrastructure of Endothia parasitica. Comparison of a Virulent with a Hypovirulent Isolate. Canadian Journal of Botany, 61, 389-399. http://dx.doi.org/10.1139/b83-046

[5] Chen, B., Chen, C.-H., Bowman, B. and Nuss, D. (1996) Phenotypic Changes Associated with Wild-Type and Mutant Hypovirus RNA Transfection of Plant Pathogenic Fungi Phylogenetically Related to Cryphonectria parasitica. Phytopathology, 86, 301-310. http://dx.doi.org/10.1094/Phyto-86-301

[6] Rigling, D. and Hillman, B.I. (2002) Genus Hypovirus (Hypoviridae). In: Tidona, C.A. and Darai, G., Eds., The Springer Index of Viruses, Springer, Berlin, New York, 456-460. http://dx.doi.org/10.1007/3-540-31042-8_71

[7] Nuss, D.L., Hillman, B.I., Rigling, D., Suzuki, N. (2005) Family Hypoviridae. In: Franquet, C.M., Mayo, M.A., Maniloff, J., Desselberger, U. and Ball, L.A., Eds., Virus Taxonomy: VIIIth Report of the International Committee for the Taxonomy of Virueses, Elsevier/Academic Press, London, 597-601. 
[8] Hillman, B.I., Halpern, B.T. and Brown, M.P. (1994) A Viral dsRNA Element of the Chestnut Blight Fungus with a Distinct Genetic Organization. Virology, 201, 241-250. http://dx.doi.org/10.1006/viro.1994.1289

[9] Hillman, B.I. and Suzuki, N. (2004) Viruses of the Chestnut Blight Fungus, Cryphonectria parasitica. Advances in Virus Research, 63, 423-472. http://dx.doi.org/10.1016/S0065-3527(04)63007-7

[10] Linder-Basso, D., Dynek, J.N. and Hillman, B.I. (2005) Genome Analysis of Cryphonectria Hypovirus 4, the Most Common Hypovirus Species in North America. Virology, 337, 192-203. http://dx.doi.org/10.1016/j.virol.2005.03.038

[11] Allemann, C., Hoegger, P., Heiniger, U. and Rigling, D. (1999) Genetic Variation of Cryphonectria Hypoviruses (CHV1) in Europe, Assessed Using RFLP Markers. Molecular Ecology, 8, 843-854. http://dx.doi.org/10.1046/j.1365-294X.1999.00639.x

[12] Robin, C., Lanz, S., Soutrenon, A. and Riglig, D. (2010) Dominance of Natural over Released Biological Control Agents of the Chestnut Blight Fungus Cryphonectria parasitica in South-Eastern France Is Associated with FitnessRelated Traits. Biological Control, 53, 55-61. http://dx.doi.org/10.1016/j.biocontrol.2009.10.013

[13] Peters, F.S., Holweg, C.L., Rigling, D. and Metzler, B. (2012.) Chestnut Blight in South-Western Germany: Multiple Introductions of Cryphonectria parasitica and Slow Hypovirus Spread. Forest Pathology, 42, 397-404. http://dx.doi.org/10.1111/j.1439-0329.2012.00773.x

[14] Chen, B. and Nuss, D. (1999) Infectious cDNA Clone of Hypovirus CHV1-Euro 7: A Comparative Virology Approach to Investigate Virus Mediated Hypovirulence of Chestnut Blight Fungus Cryphonectria parasitica. Journal of Virology, 73, 985-992.

[15] Peever, T.L., Liu, Y.C. and Milgroom, M.G. (1997) Diversity of Hypoviruses and Other Double-Stranded RNAs in Cryphonectria parasitica in North America. Phytopathology, 87, 1026-1033. http://dx.doi.org/10.1094/PHYTO.1997.87.10.1026

[16] Milgroom, M.G. and Cortesi, P. (2004) Biological Control of Chestnut Blight with Hypovirulence: A Critical Analysis. Annual Review Phytopathology, 42, 311-338. http://dx.doi.org/10.1146/annurev.phyto.42.040803.140325

[17] Hillman, B.I., Foglia, R. and Yuan, W. (2000) Satellite and Defective RNAs of Cryphonectria hypovirus 3-Grand Haven 2, a Virus Species in the Family Hypoviridae with a Single Open Reading Frame. Virology, 276, 181-189. http://dx.doi.org/10.1006/viro.2000.0548

[18] Smart, C.D., Yuan, W., Foglia, R., Nuss, D.L., Fulbright, D.W. and Hillman, B.I. (1999) Cryphonectria hypovirus 3, a Virus Species in the Family Hypoviridae with a Single Open Reading Frame. Virology, 265, 66-73. http://dx.doi.org/10.1006/viro.1999.0039

[19] Turchetti, T. and Maresi, G. (2006) Management of Diseases in Chestnut Orchards and Stands: A Significant Prospect. Advances in Horticultural Science, 20, 33-39.

[20] Turchetti, T., Ferretti, F. and Maresi, G. (2008) Natural Spread of Cryphonectria parasitica and Persistence of Hypovirulence in Three Italian Coppice Chestnut Stands. Forest Pathology, 38, 227-243. http://dx.doi.org/10.1111/j.1439-0329.2008.00557.x

[21] Anagnostakis, S.L. (1988) Cryphonectria parasitica, Cause of Chestnut Blight. Advances in Plant Pathology, 6, 123136. http://dx.doi.org/10.1016/B978-0-12-033706-4.50011-6

[22] MacDonald, W.L. and Fulbright, D.W. (1991) Biological Control of Chestnut Blight: Use and Limitations of Transmissible Hypovirulence. Plant Disease, 75, 656-660. http://dx.doi.org/10.1094/PD-75-053

[23] Anagnostakis, S.L. (1987) Chestnut Blight: The Classical Problem of an Introduced Pathogen. Mycologia, 79 , 23-37. http://dx.doi.org/10.2307/3807741

[24] Cortesi, P., McCullough, C.E., Song, H., Lin, H. and Milgroom, M.G. (2001) Genetic Control of Horizontal Virus Transmission in the Chestnut Blight Fungus, Cryphonectria parasitica. Genetics, 159,107-118.

[25] Papazova-Anakieva, I., Sotirovski, K., Cortesi, K. and Milgroom, M.G. (2008) Horizontal Transmission of Hypovirusus between Vegetative Compatibility Types of Cryphonectria parasitica in Macedonia. European Journal of Plant Pathology, 120, 35-42. http://dx.doi.org/10.1007/s10658-007-9191-z

[26] Bryner, S.F. and Rigling, D. (2012) Virulence Not Only Costs but Also Benefits the Transmission of a Fungal Virus. Evolution, 66, 2540-2250. http://dx.doi.org/10.1111/j.1558-5646.2012.01637.x

[27] Elliston, J.E. (1977) Pathogenicity and Sporulation of Normal and Diseased Strains of Endothia parasitica in American Chestnut. In: MacDonald, W.L., Cech, F.C., Luchok, J. and Smith, H.C., Eds., Proceedings of American Chestnut Symposium, West Virginia University Press, Morgantown, 95-100.

[28] Peever, T.L., Liu, Y., Cortesi, P. and Milgroom, M. (2000) Variation in Tolerance and Virulence in the Chestnut Blight Fungus-Hypovirus Interaction. Applied and Environmental Microbiology, 66, 4863-4869. http://dx.doi.org/10.1128/AEM.66.11.4863-4869.2000

[29] Bryner, S F. and Rigling, D. (2012) Hypovirus Virulence and Vegetative Incompatibility in Populations of the Chest- 
nut Blight Fungus. Phytopathology, 102, 1161-1167. http://dx.doi.org/10.1094/PHYTO-01-12-0013-R

[30] Chen, B., Geletka, L.M. and Nuss, D.L. (2000) Using Chimeric Hypoviruses to Fine-Tune the Interaction between a Pathogenic Fungus and Its Plant Host. Journal of Virology, 74, 7562-7567. http://dx.doi.org/10.1128/JVI.74.16.7562-7567.2000

[31] Liu, Y.C., Double, M.L., MacDonad, W.L. and Milgroom, M.G. (2002) Persistence of Cryphonectria Hypoviruses after Their Release for Biological Control of Chestnut Blight in West Virginia Forests. Forest Pathology, 32, 345-356. http://dx.doi.org/10.1046/j.1439-0329.2002.00299.x

[32] Elliston, J.E. (1985) Preliminary Evidence for Two Debilitating Cytoplasmic Agents in a Strain of Endothia parasitica from Western Michigan. Phytopathology, 75, 170-173. http://dx.doi.org/10.1094/Phyto-75-170

[33] Hoegger, P.J., Heiniger, U., Holdenrieder, O. and Rigling, D. (2003) Differential Transfer and Dissemination of Hypovirus and Nuclear and Mitochondrial Genomes of a Hypovirus-Infected Cryphonectria parasitic Strain after Introduction into a Natural Population. Applied and Environmental Microbiology, 69, 3767-3771. http://dx.doi.org/10.1128/AEM.69.7.3767-3771.2003

[34] McCarroll, D.R. and Thor, E. (1978) Biological Control of Chestnut Blight. In: MacDonald, W.L., Cech, F.C., Luchok, J. and Smith, H.C., Eds., Proceedings of American Chestnut Symposium, West Virginia University Press, Morgantown, 70-72.

[35] Hebard, F.V. and Shain, L. (1988) Effects of Virulent Endothia parasitica and Their Metabolites on Ethylene Production by Bark of American and Chinese Chestnut and Scarlet Oak. Phytopathology, 78, 841-845.

http://dx.doi.org/10.1094/Phyto-78-841 
Scientific Research Publishing (SCIRP) is one of the largest Open Access journal publishers. It is currently publishing more than 200 open access, online, peer-reviewed journals covering a wide range of academic disciplines. SCIRP serves the worldwide academic communities and contributes to the progress and application of science with its publication.

Other selected journals from SCIRP are listed as below. Submit your manuscript to us via either submit@scirp.org or Online Submission Portal.
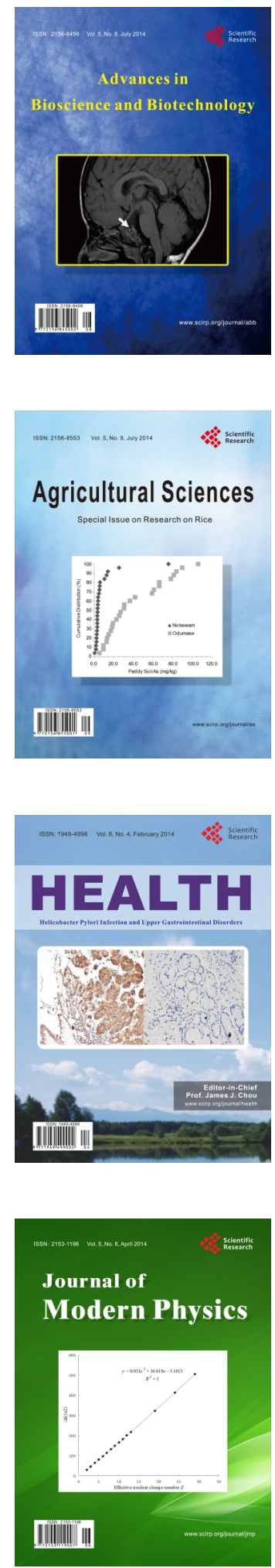
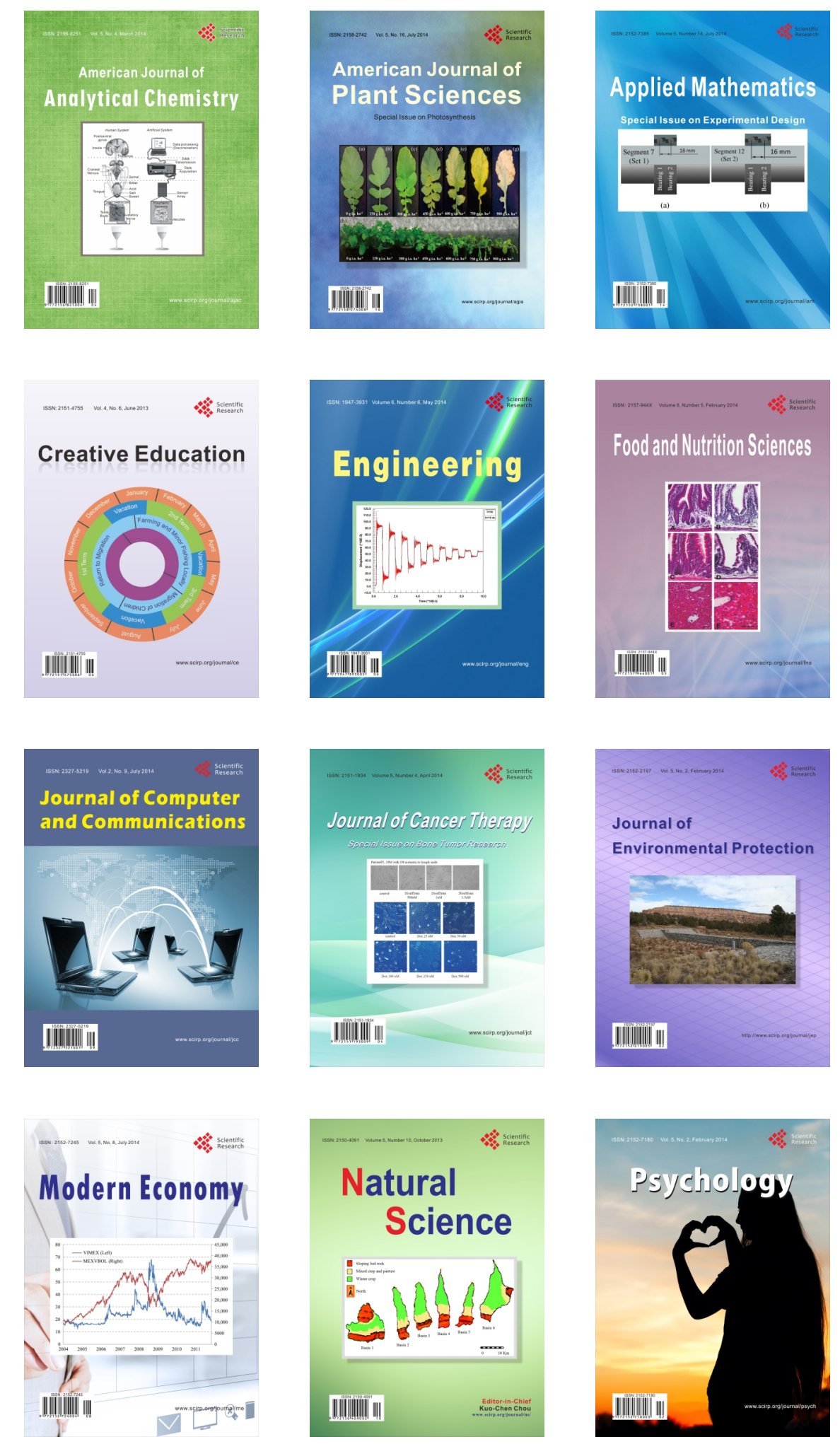